\title{
Fall Detection Methods for the Safe and independent living of Elderly People and Patients
}

\author{
Gagana $\mathbf{P}^{1}$, Vani $K \mathrm{~S}^{2}$ \\ UG Student, Department of CSE, Acharya Institute of Technology ${ }^{1}$ \\ Assistant Professor, Department of CSE, Acharya Institute of Technology ${ }^{2}$
}

\begin{abstract}
As we grow old, our desire for independence does not diminish; yet our health increasingly needs to be monitored. Injuries such as falling can be serious problem for the elderly. If a person falls and is not able to get assistance within an hour, casualties arising from the fall can result in fatalities as early as 6 months later. It would seem then that a choice between safety and independence must be made. Fortunately, as health care technology advances, simple devices can be made to detect or even predict falls in the elderly, which could easily save lives without too much intrusion on their independence. Much research has been done on the topic of fall detection and fall prediction. Some have attempted to detect falls using a variety of sensors such as cameras, accelerometers, microphones, or a combination of the same. This paper is aimed at reporting which existing methods have been found effective and the combination of which will assist in the progression towards a safe, unobtrusive monitoring system for independent living.
\end{abstract}

Keywords: Fall Detection, Kinect camera, Sensors, Elderly people.

\section{I.INTRODUCTION}

Traditionally, elderly care has been the responsibility of family members and was provided within the extended family home but in the recent years in modern societies this is decreasing. The reasons for this change include decreasing family size, the greater life expectancy of elderly people, the geographical dispersion of families, and the tendency for women to be educated and work outside the home. Falls are one of the most important health care issues for elderly people at home, old age homes and Hospitals. Fall detection system is becoming more important to elderly people and patients who will be left alone in the home because the fall may cause severe injury or even mortality. Due to the limited number of nursing staff and increasing costs of health care facilities, many research groups are focusing on the development of scientific methodologies to assist the elderly people in their homes where continuous monitoring of the elderly people and patients is required. Patient monitoring and surveillance systems based on information and communication technology are attractive for their potential to reduce the burden and cost of giving care to elderly people while maintaining safety, Privacy and autonomy. The main motivation is to minimize human involvement in surveillance and to detect more complex activities and situations using various sensors and design a novel classifier that identifies risky situations with the collected information and inform the caretakers. Robots can be used to provide the primary aid required. Therefore Automatic fall detection systems are very essential and are being developed.

A serious fall at home may lead to the risk of death or "post-fall syndrome" if the person cannot call for help immediately after a fall. In light of this, development of fall detection system has gained attention in recent years as this system can alert care takers in time after a fall has occurred in order to prevent post-fall consequences and save the life of the elderly.

Several kinds of approaches for detecting falls automatically have been proposed they can be mainly classified into three categories: wearable sensorbased, ambience device based and computer visionbased methods. Wearable sensor based methods often use specialized equipments and sensors, such as accelerators and gyroscope sensors, which are attached to the human body. When a fall occurs, it can be detected by the sensors and a help is automatically called for or be made manually via help buttons. One of the major problems is that wearable sensors are disturbing and often burdensome. Ambience device-based methods often take advantage of ambience sensors installed on the elders' active regions such as vibration sensors on the floor and IR sensors, or combine these sensors together to improve the performance.

However, some of these have a disadvantage of sensing signals of everything in and around the object by the ambiance devices, which generates false alarms and leads to a low detection accuracy. The recent advancements in computer vision technology have brought together a new solution to overcome these drawbacks. One of the main advantages of visual based fall detection is that such system does not require a person to wear any additional electronic gadget, and it is less intrusive compared to the wearable sensor. With this, visual-based home monitoring system is able to give information on falls and also other activities of daily living behaviours which are useful for home healthcare monitoring, such as medication intake, mealtime, and sleep duration. 
A combination of Sensors and Visual Surveillance techniques can lead to amore efficient monitoring of elderly people byprotecting their privacy but not compromising their independence.

\section{REASONS AND CONSEQUENCES OF ELDERLY FALL}

A fall event is one of the main factors that influence the physical and psychological health of an elderly person. Reasons for fall among elderly people include age-related vision diseases, as well as not wearing glasses that have been prescribed. Reasons for fall also include the health conditions such as Parkinson's disease, Alzheimer's disease and arthritis cause weakness in the extremities, poor grip strength, balance disorders and cognitive impairment. Injuries related to falls include physical damages like skin abrasions, bone fractures and general connective tissue lesions.

A fall has also dramatic psychological consequences, since it drastically reduces the self-confidence and independence of affected people. This may contribute to future falls with more serious outcomes or it may lead to a decline in health. Other frequent consequences include early nursing home admission and continuous fear of falling, lowering the quality of everyday life. The consequences of a fall event depend also on the long-lie period, i.e. the time interval during which the person remains involuntarily on the ground after the fall .

The severity of injury is generally related to the height of the fall. The state of the ground surface onto which the victim falls is also important, harder surfaces causing more severe injury. Meeting the challenges of elderly falls requires a clear understanding of the prevalence and nature of falls, innovative planning to develop prevention programmes, systems and structures which will support falls prevention initiatives, and substantial reforms and policies at the local and national levels. Therefore, it is of fundamental importance to provide quick support to the injured people as soon as a fall happened.

\section{III .PRODUCTS AND SOLUTIONS AVAILABLE TO DETECT FALLS}

The firstfunction of the paper is to provide a survey of different fall-detection methods.

\section{A. Microwave radar sensor}

In [1], a telehealth system aiming at remote fall detection in an in-door environment has been presented. It consists of a microwave radar sensor and a wirelessly connected base station for data processing. The implementation of the fall detection algorithm by means of a DSP platform has been presented. Experimental results conducted with human subjects under real circumstances have shown a sensitivity to detect fall events in real-time of $100 \%$, without reporting any false positives, with a maximum delay of about $316 \mathrm{~ms}$.

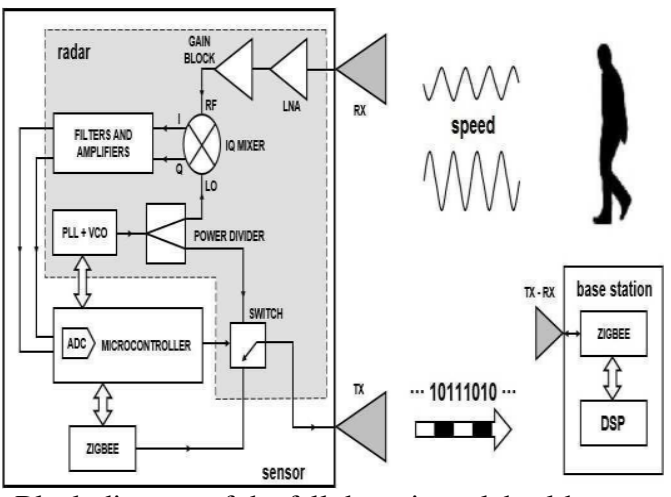

[ 1]. Block diagram of the fall detection telehealth system depicting a microwave radar and wirelessly connected base station.

The tests have been performed in an area where the radar's operation was not limited by practical situations, namely signal power, coverage of the antennas, and presence of obstacles between the subject and the antennas. Moreover, the proposed approach would allow to process at the same time up to six sensors without loss of information. This system is the result of the convergence of information, wireless technologies, and radar techniques, and is in-line with the growing need for health technologies and applications to enhance the quality-of-care for elderly people both in home and clinical environment, but the disadvantage remains that the sensors have range. They can be blocked by obstacles. The Sensor can detect only if the user is in front of it at an angle between 0 and 45 degrees.

\section{B. $\quad$ Wall mounted devices}

In [2], a wall mounted device called as the eWall is discussed. The eWALL concept is a dynamic "caring home' cognitive environment that "senses" and "learns" the needs of the corresponding primary eWALL user. It provides unobtrusive daily support, notifying informal and formal caregivers whenever necessary and serving as a bridge to supportive services offered by the outside world. The sensor devices and their intelligent interconnection in sensor networks together with novel user interfaces represent the crucial technical enablers of the eWALL assistive environment. The eWALL concept is a holistic infrastructure model that uses an interactive wallmounted device to provide a variety of context-aware closed-loop intelligent services by SOA approach. The monitoring of the living area and the body of the primary eWALL user, as well as the feedback from the system to that user is handled by the eWALL devices. The living space is monitored by cameras, microphones, furniture contact sensors, switch status sensors, environmental sensors. Monitoring information from the primary user's body is obtained by wearable devices, (e.g., a smart stethoscope that provides the decoupled lung and heart signals or accelerometers for fall detection.) Monitoring may also be performed remotely using the cameras and microphones to track the body, detect the mood of the user and understand spoken commands/requests. The input of all these devices 
is the environment and the body. Their outputs are signals to be processed by the $\mathrm{A} / \mathrm{V}$ and the non $\mathrm{A} / \mathrm{V}$ perceptual components. The system feedback is done via the main eWALL screen, the house speakers and possibly the system tablet. The input of these devices stems from the eWALL applications and their output is consumed by the elderly user. Any actuators included in the home environment use the input from the eWALL applications. Their output is the control of the different home appliances. All the eWALL devices are connected to the devicegateway. The device gateway is the eWall gateway component connecting, configuring, querying and reporting data from sensor/actuators and $\mathrm{A} / \mathrm{V}$ devices. This component acts as an aggregator of sensor measurement data and $\mathrm{A} / \mathrm{V}$ streams, and is a unified gateway to the other components of the eWall home system. The eWALL sensor devices are very versatile. They span from medical sensors (that measure pulse rate, ECG, body core temperature, breathing rate, blood pressure, oxygen saturation, glucose etc.) to home safety and smart home sensors and actuators (that measure light, ambient temperature, CO2 level, facial expression presence of other people, motion etc.).

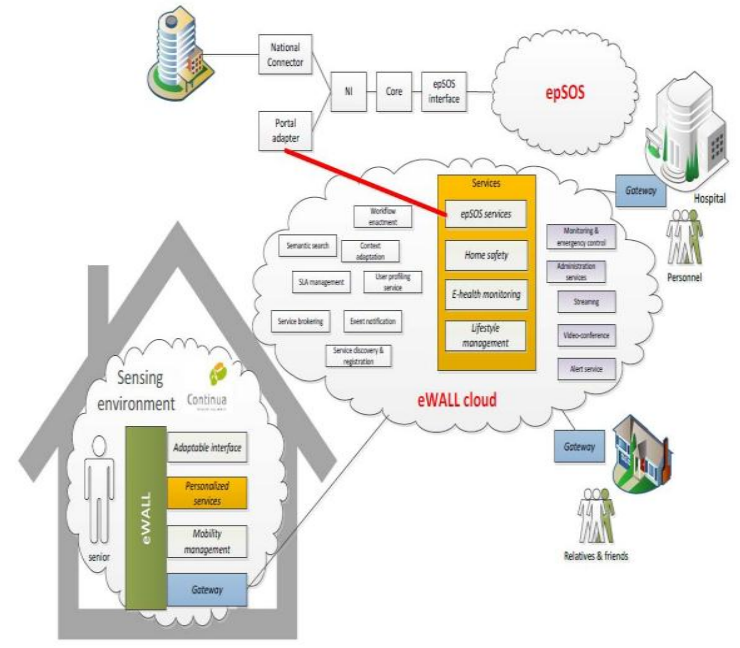

[2] Fig. depicts eWALL holistic service oriented architectureconsisting of a sensing environment, eWall cloud and several gateways.

One of the cornerstone novelties of the eWALL platform, is the use of smart and intelligent functionalities to integrate the eWALL devices, thus creating an innovative autonomous sensor network platform, which performs advanced and adaptable sensing. This paper presented the conceptual framework of an ambient assisted living service platform to elderly and cognitively or chronically impaired users that offers a constant monitoring and interpretation of health-related parameters in the user's everyday life, leading to reduction in visits to doctors or time spent for hospitalization. The eWALL platform intelligently interacts the latest ICT technologies to provide a number of services and applications that have the potential to improve the quality of life, and prolong longevity. eWALL has the potential to increase the independent living of the elderly for an average of two years, which can be translated into a significant social and economic impact. eWALL will have a flexible open API to enable integration with a number of different technologies. Even after having so many advantages there are vital areas in which eWall fails. The primary users may forget to wear the body mounted devices. Also inclusion of audio and video surveillance steals away privacy.

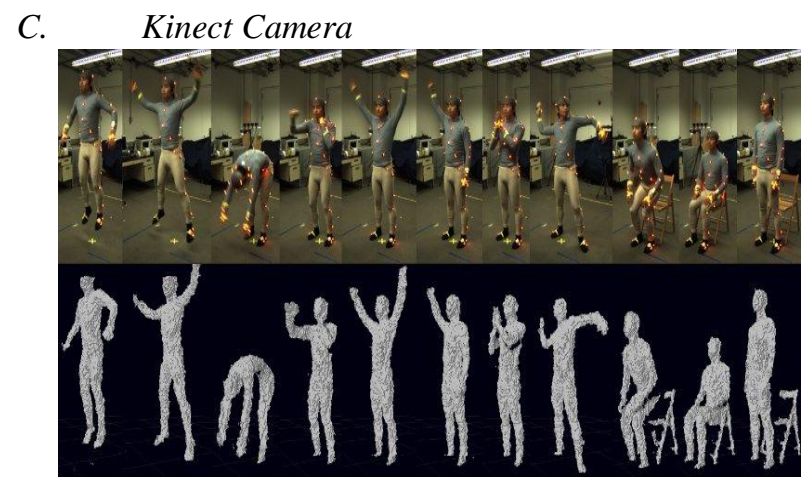

[3]. Fig. depicts extraction of background and gathering mobile points using a Kinect camera.

In [3], a RGB-Depth Camera called as the Kinect camera is discussed in order to detect falls and the activity of elderly people. Our algorithm extract the background with method "running average" to obtain only mobile pixels. After we gather these mobile pixels belonging to the person. Thus we can track this person and more precisely we track her centre of mass. In this paper we want to identify which activity the person made. For distinguishing several behaviours we create a HMM with eight states corresponding to eight situations of a daily life. We realize an experiment to verify the accuracy of the model. The results show that the model provide a good classification of the situations. The algorithm detect the falls without false positives. The algorithm can also distinguish between a person sitting, walking, squatting, going up, lying on a couch and lying down.

\section{Wireless Sensor Data}

Paper [4] sheds light on wearable sensors. They have been designed to understand human postures, RFID markers tracking patients, wearable accelerometers detecting falls, etc. Wearable sensors, however, are not always convenient for users; particularly where long term monitoring is required.

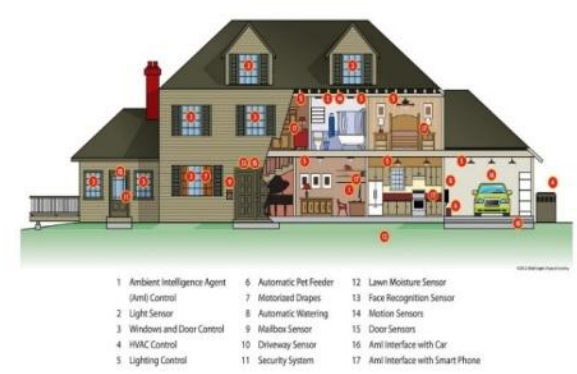

[4]. Fig. depicts various wireless sensors developed for a smarter and safer homes. 
Motivated by these limitations, they developed a Smarter Safer Homes platform that enables an automated approach of assessing fundamental ADLs of independent living of older people through information gathered from wireless sensors placed within their home environment.

This platform thus provides longitudinal objective information about the resident's day-to-day activity status, and therefore can assist self-management of residents or timely care attended from family member or carers. To achieve this, data gathered from these sensors will be used collectively to determine the residents' actions through analysis of various context-related actions through the devised platforms human behaviour detection algorithms to automatically infer health related activities pertaining to ADLs.

These sensors communicate in-home activity data with a local server through the ZigBee protocol that enables lowpower, secure and reliable data transmission. Motion sensors detect the presence of people in its vicinity. They are installed in every room to monitor the location and transition within the home.

Accelerometer sensors are attached to beds to monitor status transfers from lying/standing and sleep quality through measurement of subtle body movements in bed.

Power sensors are plugged into power outlets to measure electrical power consumption of connected appliances. Acoustic sensors are attached to downpipes under the kitchen basin to detect sound generated by water flowing in pipes.

A combined temperature/humidity sensor installed in the bathroom and kitchen to detect the changes in room air condition. Reed switches affixed to door contact surfaces detect opening/closing of the entrance door, wardrobes, cupboards, etc. And finally circuit meter sensors installed in the meter box monitor energy usage from kitchen stoves. Most of the environmental sensors are powered by batteries.

This makes sensor installations flexible and easy because it is untethered and can be positioned less intrusively and yet close to the activity being gathered, independent of a power source. It also benefits easy sensor maintenance. Furthermore the sensor communication generally requires little bandwidth and is relatively insensitive to latency, so that we can apply energy efficient communication protocols and event-based communication strategies, i.e. only uploading sensor data whenever an event has been detected. In this way, the sensors battery life isgreatly extended lasting an average six to eight months long. Although the battery needs to be changed periodically.

\section{E.On Body Sensors}

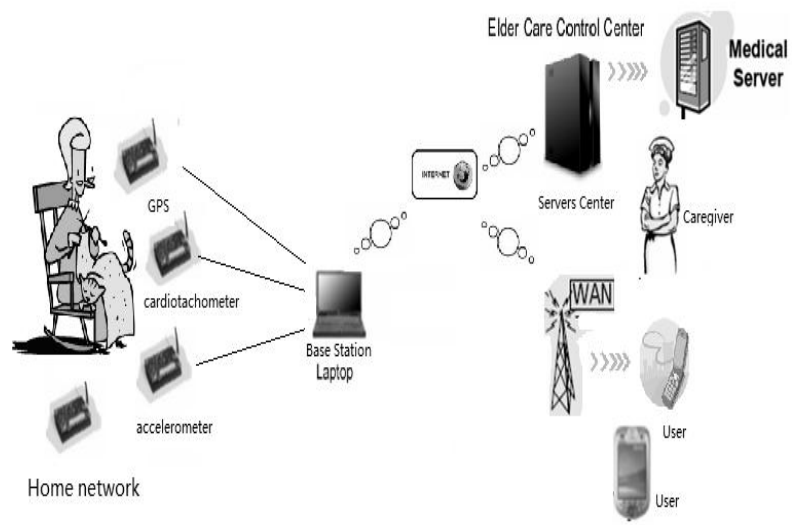

[5]. Fig. depicts various wireless sensors along with a base station and control centres

In [5], an enhanced fall detection system based on Onbody smart sensors is proposed, implemented, anddeployed that successfully detects accidental falls in consumer home application. By using information from anaccelerometer, smart sensor and cardiotachometer, the impactsof falls can successfully be distinguished from activities of daily lives reducing the false detection of falls. From thedataset of 30 participants, it is found that the proposed falldetection system achieved a high accuracy of $97.5 \%$, and thesensitivity and specificity are $96.8 \%$ and $98.1 \%$ respectfully.The proposed system is ready to be implemented in a consumer device, But the drawback remains that the system is implemented on wearable devices which makes detecting falls impossible when the user forgets to wear them.

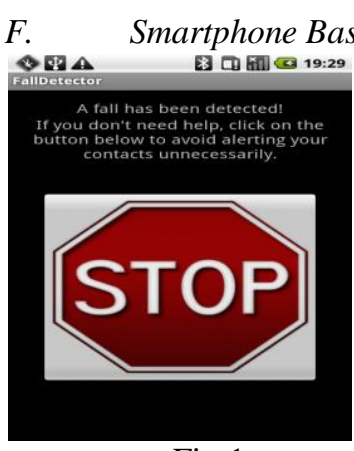

Fig 1

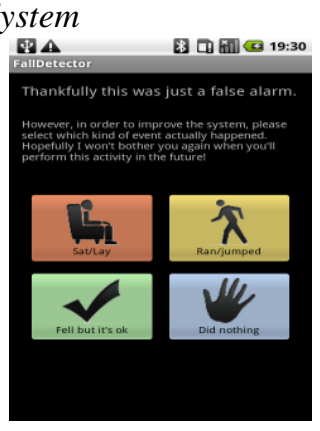

Fig 2
[6]. Figure 1 and 2 displays the notification window of a smartphone

In [6] we discuss about asmartphone based fall detection system.The contribution of the work presented in this paper to the body of existing literature is twofold. First, it shows that the recognition of fall-like activities of daily living (ADL), on the basis of peculiar features of the acceleration patterns, can significantly reduce the number of false alarms. It is important to note that, in a trivial system where an alarm is raised when acceleration becomes higher than the $3 \mathrm{~g}$ threshold, all the fall-like 
ADLs in our dataset would produce a false alarm. Second, the interviews with the group of aged people showed that usability is a fundamental factor for a real adoption of a fall detection system. In particular, forcing the users to place the phone on their belt can make a fall detection system unattractive for a large part of the population. In this context, the possibility of using a small external sensing unit can greatly reduce the intrusiveness of the system. However the smartphones needs to be recharged time and again, also it is impractical for a user to press the help button during a sudden fall.The extension of the same smartphone technology can be seen in $[7,8]$. Along with that, it also uses Global positioning system to pinpoint and notify the user's exact location to the caretakers in case of an emergency.

\section{CONCLUSION}

Over the broad spectrum of previous works used in fall detection, the most feasible solution seems to be a combination of multiple Kinect cameras and wireless sensor network in the form of multiple activity detection sensors. There is an obvious increase in efficiency by using the duo, but the main concern stays with faster drain of resources in the form of battery life. Hence we propose a solution wherein the Kinect cameras shall be activated only when the activity detection system is serene for a longer period of time.

\section{SUMMARY}

\begin{tabular}{|c|c|c|}
\hline Name & Title & Algorithms and sensors used \\
\hline $\begin{array}{l}\text { Carmine Garripoli et. } \\
\text { al }\end{array}$ & $\begin{array}{l}\text { Embedded DSP-based Telehealth Radar System } \\
\text { for Remote In-door Fall Detection }\end{array}$ & $\begin{array}{l}\text { Digital Signal Processing, Radar } \\
\text { sensor }\end{array}$ \\
\hline AlbenaMihovska et. al & $\begin{array}{l}\text { eWall for Active Long Living: } \\
\text { Assistive ICT Services for Chronically Ill and } \\
\text { Elderly Citizens }\end{array}$ & $\begin{array}{l}\text { Body sensors, Ontological } \\
\text { sensors, } \\
\text { Medical sensors }\end{array}$ \\
\hline Amandine Dubois et al & $\begin{array}{l}\text { Human Activities Recognition } \\
\text { with RGB-Depth Camera using HMM }\end{array}$ & $\begin{array}{l}\text { RGB-depth camera, Forward- } \\
\text { Backward algorithm }\end{array}$ \\
\hline Qing Zhang et al & $\begin{array}{l}\text { Activity of Daily Living Assessment through } \\
\text { Wireless Sensor Data }\end{array}$ & $\begin{array}{l}\text { Motion sensor, Power sensor, } \\
\text { Acoustic sensor, Reed switches, } \\
\text { Humidity sensor, Temperature } \\
\text { sensors }\end{array}$ \\
\hline Jin Wang et al & $\begin{array}{l}\text { An Enhanced Fall Detection System } \\
\text { for Elderly Person Monitoring } \\
\text { using Consumer Home Networks }\end{array}$ & Cardiotachometer, Accelerometer \\
\hline Stefano Abbatea et al & A smartphone-based fall detection system & $\begin{array}{l}\text { Acceleration threshold algorithm, } \\
\text { Back- } \\
\text { propogationalgorithm, velocity } \\
\text { estimation algorithm }\end{array}$ \\
\hline Jiangpeng Dai et al & Mobile phone-based pervasive fall detection & $\begin{array}{l}\text { Acceleration algorithm, shape } \\
\text { context and hausdorff distance } \\
\text { algorithm, Accelerometer sensor, } \\
\text { Orientation sensor, Digital } \\
\text { magnetic field sensor }\end{array}$ \\
\hline $\begin{array}{l}\text { GokhanRemziYavuz } \\
\text { et. al }\end{array}$ & $\begin{array}{l}\text { A Smartphone Based Fall Detector with Online } \\
\text { Location Support }\end{array}$ & $\begin{array}{l}\text { Thresholding algorithm, Acoustic } \\
\text { sensors, Accelerometer }\end{array}$ \\
\hline
\end{tabular}

\section{REFERENCES}

1. Carmine Garripoli, Marco Mercuri, et.al Embedded DSP-based Telehealth Radar Systemfor Remote In-door Fall Detection, March 2014.

2. AlbenaMihovska, Sofoklis A. Kyriazakos, and Ramjee Prasad, eWall for Active Long Living: Assistive ICT Services for chronically ill and services citizens.

3. Amandine Dubois and François Charpillet, Human Activities Recognition with RGBDepth Camera using HMM, July, 2013.

4. Qing Zhang,Mohan Karunanithi,Dana Bradford,Yasmin van Kasteren, Activity of Daily Living Assessment through Wireless Sensor Data, 2014.

5. Jin Wang, An Enhanced Fall Detection System for Elderly Person Monitoring using Consumer Home Networks, IEEE, 2014
6. Stefano Abbatea, Marco Avvenutia, Francesco Bonatesta, GuglielmoColaa, Paolo Corsinia, AlessioVecchioa , A smartphone-based fall detection system, University of Pisa , 2012

7. JiangpengDai ,Xiaole Bai ,Zhimin Yang ,ZhaohuiShen ,Dong Xuan , Mobile phone-based pervasive fall detection , SpringerVerlag London Limited, 2010.

8. GokhanRemziYavuz, Mustafa ErayKocak, Gokberk Ergun, HandeAlemdar, Hulya Yalcin,OzlemDurmazIncel, LaleAkarun, CemErsoy, A Smartphone Based Fall Detector with OnlineLocation Support 